\title{
Folate receptor-mediated cervical staining as an adjunct to colposcopy which can improve the diagnostic accuracy of detecting high grade squamous intraepithelial lesions
}

\author{
Wojciech Homola@, Michal Pomorski®, Aleksandra Zimmer®D, Pawel Baranski®, Mariusz Zimmer® \\ $2^{\text {nd }}$ Department and Clinic of Gynaecology, Obstetrics and Neonatology, Wroclaw Medical University, Wroclaw, Poland
}

\begin{abstract}
Objectives: Cervical cancer is rated fourth in terms of incidence and cancer-related mortality in women. Cytology-based screening programs and colposcopy provided insufficient rates of detecting cervical intraepithelial neoplasia (CIN) prompting researchers to develop new tools. The aim of this study was to evaluate whether folate receptor-mediated staining is useful in detecting CIN2+ during gynecological examination with colposcopy.

Material and methods: In total 96 women with abnormal cytology findings were enrolled. The study was conducted on the Polish population. The diagnostic process consisted of colposcopy, receptor-mediated diagnosis (FRD), and histopathology examination. All women were subjected to the same diagnostic procedure.

Results: The patient mean age of 96 women was $38 \pm 14.5$ years. On colposcopy, high-grade lesions were detected in 83 women. The FRD gave positive results in 63 women. Histopathology revealed 1 case of carcinoma plano epithelial akeratodes, 21 cases of high-grade squamous intraepithelial lesions, 13 cases of low-grade squamous intraepithelial lesions. A total of 61 cases presented no pathology. FRD as an adjunct to colposcopy gave the following test results in detecting CIN2+ lesions: sensitivity $-94.29 \%$, specificity $-46.67 \%$, PPV $-50.77 \%$, NPV $-93.33 \%$, and accuracy $-64.21 \%$. Using both techniques provided better results than using each of the tests alone.

Conclusions: $\mathrm{FRD}$ is a promising test for the diagnosing CIN2+ cervical pathologies because it can increase the probability of detecting $\mathrm{CIN} 2+$ without any additional burden posed on patients. Further studies should be conducted on large and various populations to complement current evidence.
\end{abstract}

Key words: cervical neoplasia; cervical cancer; folate receptor-mediated cervical staining; FRD

Ginekologia Polska 2020; 91, 5: 247-250

\section{INTRODUCTION}

Cervical cancer is a considerable problem for women's health. The estimated number of new cancer cases reached 569,800 , and the estimated number of deaths was 311,400 in 2018 worldwide, which placed cervical cancer at the fourth position in terms of incidence and cancer-related mortality in women [1]. Increasing awareness and introduction of screening programs allowed to decrease morbidity and mortality rates due to cervical cancer [2]. In high-income countries, indices for cervical cancer are much lower than in low-income countries. For this reason, cervical screening is one out of three elements of the WHO global strategy towards eliminating cervical cancer worldwide as a public health problem [3].
Currently, many screening programs are based on cytology [4]. However, this method is considered to be of insufficient and differentiated accuracy. An 11-year retrospective analysis of 999 cases published by Kang et al. reported a sensitivity of $97.14 \%$ and specificity of $85.58 \%$ for detecting high-grade squamous intraepithelial lesion (HSIL) and squamous cell carcinoma (SCC) [5]. But the study by Wojciech et al. [6] on patients with histologically confirmed cervical intraepithelial neoplasia (CIN) showed a sensitivity of $58.02 \%$ and specificity of $63.28 \%$ in detecting CIN. For this reason, other noninvasive methods such as based on electrical impedance spectroscopy, folic acid receptor-mediated diagnosis (FRD) method, or those employing arti- 
ficial intelligence are of great interest [7-9]. The method based on FRD was developed after it had been discovered that the expression of the folate receptor $a$ is increased not only in certain cells but also in many epithelial cancers [10]. Despite the FRD staining being a new method, the evidence is growing rapidly. Preliminary results show that this method is distinguished by simplicity, rapid provision of results, and effectiveness. Therefore, we undertook this study to add evidence on FRD. The aim of this study was to evaluate whether folate receptor-mediated staining is useful in detecting CIN2+ during gynecological examination with colposcopy.

\section{MATERIAL AND METHODS}

Women with abnormal cytology findings were enrolled in this study. All women were referred to our institution - an outpatient colposcopy clinic in a tertiary gynecology and obstetrics department. The study was conducted in the Department of Gynecology and Obstetrics, Wrocław Medical University, Wroclaw, Poland. Women who suffered from cancer of the cervix, had a vaginal bleeding or active menstruation, had used vaginal contraceptives and vaginal medications up to 2 days before the enrollment were not included. All women gave the written informed consent for participation in the study and taking part in the diagnostic process to confirm or exclude CIN2+. The study was approved by the Commission of Bioethics at Wroclaw Medical University.

The diagnostic process consisted of colposcopy, FRD, and histopathology examination. Two experienced gynecologists were responsible for gynecology examinations and collection of specimens and data. One histopathologist with experience in the assessment of cervical pathology reviewed and interpreted biopsies. All study participants underwent the same diagnostic process.

For the conduct of colposcopy, the Videocolposcope HD-1000 with the IRIS software (Medicom, Wroclaw, Poland) was used. Images of the cervix were video recorded, both before and after the application of $3 \%$ acetic acid. FRD staining was done before visual inspection with acetic acid (VIA). For staining, the folate receptor-mediated staining solution (Shaanxi Gaoyuan Medical Equipment Service Co., Ltd., Shaanxi, China) was used. A single-use foam applicator soaked in the FRD solution was placed on the cervix for 30 seconds and next, in the FRD colour changes on the applicator were assessed in comparison with FRD colour changes sheet. Results were classified as CIN2+ negative: for brown or green colors, while CIN2+ or worse for blue, dark blue or black. At each examination, 2-3 targeted biopsies from the most suspected areas were taken. When there were no FRD induced colors changes, random biopsies were taken.
In the overall assessment, the case was considered to be negative for High-Grade Squamous Intraepithelial Lesions (HG-SIL) when the result of colposcopy was normal without any acetowhitening changes or any worse abnormalities; the FRD staining was brown or green, and histopathology samples were negative for HG-SIL (below CIN II).

Collected data were statistically analyzed. Categorical variables were presented as numbers and percentages, while continuous data were presented as means with a standard deviation. Results between the two diagnostic methods (classical colposcopy and colposcopy + FRD staining) were compared with the Fisher's exact test. To evaluate diagnostic tests, the following results were calculated: sensitivity, specificity, positive predictive value (PPV), negative predictive value (NPV), and accuracy.

\section{RESULTS}

Overall, 96 women were eligible for the study. The patient population mean age was 38 years old with an SD of 14.5 and range from 24 to 86 years. All study participants had abnormal cytology results which are summarized in Table 1. On colposcopy, high-grade lesions were detected in 83 women. The FRD gave positive results in 63 women. Table 2 shows the results of the histopathological examination.

Positive and negative results of colposcopy, FRD and histopathology (Tab. 3) were used to calculate sensitivity, PPV, NPV, and accuracy (Tab. 4).

\begin{tabular}{|l|l|l|}
\multicolumn{3}{|c|}{ Table 1. Cytology results of referred women ( $n=96)$} \\
\hline & Cytology diagnosis & $\begin{array}{l}\text { No of } \\
\text { patients }\end{array}$ \\
\hline 1. & Squamous cell carcinoma (SCC) & 1 \\
\hline 2. & Atypical squamous cells cannot exclude HSIL (ASC-H) & 14 \\
\hline 3. & AGC-US & 6 \\
\hline 4. & $\begin{array}{l}\text { Atypical squamous cells of undetermined } \\
\text { significance (ASC-US) }\end{array}$ & 18 \\
\hline 5. & High-grade squamous intraepithelial lesion (HSIL) & 15 \\
\hline 6. & AIS & 1 \\
\hline 7. & Low-grade squamous intraepithelial lesions (LSIL) & 36 \\
\hline 8. & $\begin{array}{l}\text { Negative for intraepithelial lesion or malignancy } \\
\text { (NILM) }\end{array}$ & 5 \\
\hline
\end{tabular}

Table 2. Histopathological results of the study group $(n=96)$

\begin{tabular}{|l|l|l|}
\hline Lp. & Histological diagnosis & $\begin{array}{l}\text { No of } \\
\text { patients }\end{array}$ \\
\hline 1. & Carcinoma plano epithelial akeratodes (G1) & 1 \\
\hline 2. & High-grade squamous intraepithelial lesion (HSIL) & 21 \\
\hline 4. & Low-grade squamous intraepithelial lesion (LSIL) & 13 \\
\hline 5. & Normal & 61 \\
\hline
\end{tabular}




\begin{tabular}{l|l|l|l|l|}
\multicolumn{3}{|l}{ Table 3. Test calculations } \\
\multirow{2}{*}{ Results of the test } & \multicolumn{3}{l}{ Histopathology results } \\
\cline { 2 - 4 } & Positive & Negative \\
\hline \multirow{2}{*}{ Colposcopy } & Positive & $n=13$ & $n=0$ \\
\cline { 2 - 4 } & Negative & $n=22$ & $n=61$ \\
\hline FRD & Positive & $n=30$ & $n=33$ \\
\hline Colposcopy & Negative & $n=5$ & $n=28$ \\
+ FRD & Positive & $n=33$ & $n=32$ \\
\hline & Negative & $n=3$ & $n=28$
\end{tabular}

\begin{tabular}{|c|c|c|c|}
\hline & Colposcopy & FRD & $\begin{array}{l}\text { Colposcopy + } \\
\text { FRD }\end{array}$ \\
\hline Sensitivity & $\begin{array}{l}37.14 \% \text { (95\% Cl: } \\
21.47-55.08)\end{array}$ & $\begin{array}{l}85.71 \%(95 \% \mathrm{Cl}: \\
69.74-95.19)\end{array}$ & $\begin{array}{l}94.29 \%(95 \% \mathrm{Cl}: \\
80.84 \%-99.30)\end{array}$ \\
\hline Specificity & $\begin{array}{l}100.00 \%(95 \% \mathrm{Cl}: \\
94.13-100.00)\end{array}$ & $\begin{array}{l}45.90 \% \text { (95\% Cl: } \\
33.06-59.15)\end{array}$ & $\begin{array}{l}46.67 \%(95 \% \mathrm{Cl}: \\
33.67-60.00)\end{array}$ \\
\hline $\begin{array}{l}\text { Positive } \\
\text { Predictive } \\
\text { Value }\end{array}$ & $100.00 \%$ & $\begin{array}{l}47.62 \%(95 \% \mathrm{Cl}: \\
41.02-54.30)\end{array}$ & $\begin{array}{l}50.77 \%(95 \% \mathrm{Cl}: \\
44.53-56.98)\end{array}$ \\
\hline $\begin{array}{l}\text { Negative } \\
\text { Predictive } \\
\text { Value }\end{array}$ & $\begin{array}{l}73.49 \%(95 \% \mathrm{Cl}: \\
68.25-78.15)\end{array}$ & $\begin{array}{l}84.85 \% \text { (95\% Cl: } \\
70.41-92.95)\end{array}$ & $\begin{array}{l}93.33 \%(95 \% \mathrm{Cl}: \\
78.01-98.22)\end{array}$ \\
\hline Accuracy & $\begin{array}{l}77.08 \% \text { (95\% Cl: } \\
67.39-85.05)\end{array}$ & $\begin{array}{l}60.42 \% \text { (95\% Cl: } \\
49.92-70.25)\end{array}$ & $\begin{array}{l}64.21 \%(95 \% \mathrm{Cl}: \\
53.72-73.79)\end{array}$ \\
\hline
\end{tabular}

$\mathrm{Cl}-$ confidence interval

\section{DISCUSSION}

Our study showed that colposcopies with an adjunct FRD have a sensitivity of $94.29 \%$, specificity of $46.67 \%$, PPV of $50.77 \%$ and NPV of $93.33 \%$. Using both techniques leads to posing less destress on patients that would have a false-positive result with a colposcopy alone and limiting further unnecessary testing. Adding FRD to colposcopies elevates sensitivity of the examination from $37.14 \%$ to $94.29 \%$ increasing the probability that a test result will be positive when the disease is present. Our results may differ from those obtained in other studies because the study group consisted of women with abnormal cytology result referred to a tertiary diagnostic center which specializes in diagnosis and treatment of more complex conditions.

FRD is a novel technique employed in oncology. It is based on the discovery that the folate receptor (FR) expression is highly elevated in certain cells, including a variety of cancers [11]. Parker at al. [12] found positive expression of FR in certain types of ovarian carcinomas as well as in kidney, endometrium, lung, breast, bladder, and pancreatic cancers. Also, normal human tissues showed to have variability in FR expression. Normal human ovaries had a negligible expression of FR while this expression was high in normal human lung tissue. Liu et al. [13] investigated the expression of FRa and the role of FRa in the regulation of the ERK signaling pathway. They found that FRa expression was progressively increasing along with the progression of cervical lesions. In squamous cell carcinoma of the cervix, expression of proteins of ERK signaling pathway correlated with the expression of FRa. They concluded that expression of FRa is associated with the progression of cervical cancer and can regulate cervical cancer cells growth.

Since the discovery of the link between FR and cervical cancer, evidence on the potential role of FR in the detection of cervical metaplasia is growing rapidly. Several studies conducted in the real clinical practice settings were published recently. Lu et al. [14] conducted a study on 169 women and compared the results of FRD and cytology testing. They reported a sensitivity of $71.93 \%$, specificity of $66.07 \%$, PPV of $51.90 \%$, and NPV of $82.22 \%$ of FRD in the diagnosis of cervical cancer and considered this result to be comparable to cytology. Li et al. [15] conducted the largest study up until now. They recruited 14,344 women from rural areas of China. In detecting CIN2+, FRD showed a sensitivity of $85.7 \%$, specificity of $76.4 \%$, PPV of $61.3 \%$, and NPV of $92.5 \%$. Authors concluded that FRD had a moderate agreement with cytology in detecting atypical squamous cells, was unable to exclude high-grade intraepithelial lesions, but was more sensitive than cytology. Xiao et al. [16] examined 404 women using FRD to screen them for high-grade cervical lesions. They found that the sensitivity of FRD in detecting CIN2+ was $80.00 \%$, specificity was $51.92 \%$, PPV was $24.19 \%$ and NPV 93.12\%. Dai et al. [17] included 216 women and subjected to FRD, human papillomavirus testing and ThinPrep cytology test. They reported the following test results for FRD: sensitivity $-80.41 \%$, specificity - 68.29\%, PPV - 60\%, NPV - 85.5\%. They concluded that FRD had significantly higher specificity than HPV testing and TCT, but no differences were noted in specificity. The recent study of Zhao et al. [18] recruited 1,504 patients with abnormal cytology and/or positive human papillomavirus (HPV) testing at primary screening. In this study, the sensitivity of FRD was $77.72 \%$ and specificity of FRD was $60.02 \%$. It is worth noting that the rate of detection of pathological lesions increased with the greater severity of the disease. FRD detected $45.45 \%$ of CIN1, $66.93 \%$ of $\mathrm{CIN} 2,84.44 \%$ of CIN3, and $98 \%$ of carcinomas.

The results of our study are in line with the above-discussed reports from the literature. The advantage of our study lies in the evaluation of the benefit of FRD as an added value to the standard of care diagnosis in our institution, while most studies focus on presenting results of FRD alone in comparison to other diagnostic methods. Nevertheless, further studies should be conducted to further investigate the usefulness of FRD in detecting CIN2+ in clinical practice 
settings. FRD is a simple technique with a rapid result which should be considered when adding this examination to the current standard of care.

\section{CONCLUSIONS}

FRD is a promising test for the diagnosing CIN2+ cervical pathologies because it can increase the probability of detecting CIN2+ without any additional burden posed on patients. Further studies should be conducted on large and various populations to complement current evidence.

\section{REFERENCES}

1. Ferlay J, Colombet $\mathrm{M}$, Soerjomataram $\mathrm{l}$, et al. Estimating the global cancer incidence and mortality in 2018: GLOBOCAN sources and methods. Int J Cancer. 2019; 144(8): 1941-1953, doi: 10.1002/ijc.31937, indexed in Pubmed: 30350310.

2. Bray F, Ferlay J, Soerjomataram I, et al. Global cancer statistics 2018: GLOBOCAN estimates of incidence and mortality worldwide for 36 cancers in 185 countries. CA Cancer J Clin. 2018; 68(6): 394-424, doi: 10.3322/caac.21492, indexed in Pubmed: 30207593.

3. Canfell K, Kim JJ, Brisson M, et al. Mortality impact of achieving WHO cervical cancer elimination targets: a comparative modelling analysis in 78 low-income and lower-middle-income countries. Lancet. 2020; 395(10224): 591-603, doi: 10.1016/S0140-6736(20)30157-4, indexed in Pubmed: 32007142.

4. Lees BF, Erickson BK, Huh WK. Cervical cancer screening: evidence behind the guidelines. Am J Obstet Gynecol. 2016; 214(4): 438-443, doi: 10.1016/j.ajog.2015.10.147, indexed in Pubmed: 26519782.

5. Kang M, Ha SY, Cho HY, et al. Comparison of papanicolaou smear and human papillomavirus (HPV) test as cervical screening tools: can we rely on HPV test alone as a screening method? An 11-year retrospective experience at a single institution. J Pathol Transl Med. 2020; 54(1): 112-118, doi: 10.4132/jptm.2019.11.29, indexed in Pubmed: 31964113.

6. Wojciech R. [The diagnostic value of cytology and colposcopy in women with cervical intraepithelial neoplasia]. Ginekol Pol. 2011; 82(8):607-611, indexed in Pubmed: 21957606.

7. Homola W, Fuchs T, Baranski $P$, et al. Use of electrical impedance spectroscopy as an adjunct to colposcopy in a pathway of cervical intraepi- thelial neoplasia diagnostics. Ginekol Pol. 2019; 90(11): 628-632, doi: 10.5603/GP.2019.0107, indexed in Pubmed: 31802462.

8. Hu L, Bell D, Antani S, et al. An Observational Study of Deep Learning and Automated Evaluation of Cervical Images for Cancer Screening. J Natl Cancer Inst. 2019; 111(9): 923-932, doi: 10.1093/jnci/djy225, indexed in Pubmed: 30629194.

9. Li K, Cai H, Shen H. Application of FRD Epithelial Tissue Special Staining Solution in Screening of Cervical Lesions. Journal of Guangxi Agricultural and Biological Science. 2016; 7: 1584-1588.

10. Lewis CM, Smith AK, Kamen BA. Receptor-mediated folate uptake is positively regulated by disruption of the actin cytoskeleton. Cancer Res. 1998; 58(14): 2952-2956, indexed in Pubmed: 9679952.

11. Carron PMc, Crowley A, O'Shea D, et al. Targeting the Folate Receptor: Improving Efficacy in Inorganic Medicinal Chemistry. Curr Med Chem. 2018; 25(23): 2675-2708, doi: 10.2174/0929867325666180209143715, indexed in Pubmed: 29424300.

12. Parker N, Turk MJo, Westrick E, et al. Folate receptor expression in carcinomas and normal tissues determined by a quantitative radioligand binding assay. Anal Biochem. 2005; 338(2): 284-293, doi: 10.1016/j. ab.2004.12.026, indexed in Pubmed: 15745749.

13. Liu C, Ding L, Bai L, et al. Folate receptor alpha is associated with cervical carcinogenesis and regulates cervical cancer cells growth by activating ERK1/2/c-Fos/c-Jun. Biochem Biophys Res Commun. 2017; 491(4): 10831091, doi: 10.1016/j.bbrc.2017.08.015, indexed in Pubmed: 28782518.

14. Lu MH, Hu LY, Du XX, et al. An special epithelial staining agents: folic acid receptor-mediated diagnosis (FRD) effectively and conveniently screen patients with cervical cancer. Int J Clin Exp Med. 2015; 8(5): 7830-7836, indexed in Pubmed: 26221336.

15. Li D, Chen L, Wang $H$, et al. Clinical application of a rapid cervical cancer screening method: Folate receptor-mediated staining of cervical neoplastic epithelia. Asia Pac J Clin Oncol. 2017; 13(1): 44-52, doi: 10.1111/ajco.12573, indexed in Pubmed: 27739250.

16. Xiao $\mathrm{S}, \mathrm{Xie} H$, Zhu X, et al. Study on the Significance of Folate Receptor-Mediated Staining Solution (FRD) Staining in Screening High Grade Cervical Lesions. Med Sci Monit. 2019; 25: 2792-2801, doi: 10.12659/MSM.911402, indexed in Pubmed: 30990212.

17. Dai Y, Wang L, Li D. Effectiveness of novel folate receptor-mediated staining solution detection (FRD) for cervical cancer screening. Medicine (Baltimore). 2018; 97(34): e11868, doi: 10.1097/MD.0000000000011868, indexed in Pubmed: 30142780.

18. Zhao Y, Li M, Li Y, et al. Evaluation of Folate Receptor-Mediated Cervical Dyeing as a Method for Detection of Cervical Lesions. J Low Genit Tract Dis. 2019; 23(2): 133-137, doi: 10.1097/LGT.0000000000000411, indexed in Pubmed: 30817686. 